\title{
Data-driven Tetrahedral Mesh Subdivision
}

\author{
Lyudmila Rodríguez, Isabel Navazo, Àlvar Vinacua ${ }^{1}$ \\ Email: $\{$ lyudmila, isabel, alvar\}@lsi.upc.es \\ Universitat Politècnica de Catalunya \\ Departament de Llenguatges i Sistemes Informatics \\ Barcelona, España \\ Abril, 2006
}

${ }^{1}$ This work was partially supported by project TIC-2000-1009 funded by the Spanish Ministry of Science and Technology 


\section{Contents}

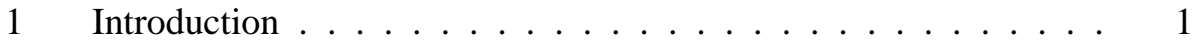

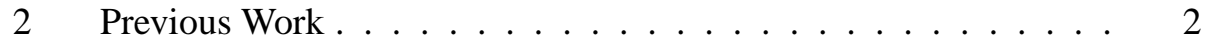

3 Our proposal ................... 5

3.1 On the computation of discrepancies within a tetrahedron . 5

3.2 Subdivision scheme . . . . . . . . . . . . 6

4 Implementation details . . . . . . . . . . . . . . . . . 15

5 Experimental Results . . . . . . . . . . . . . . . . . . . 19

$5.1 \quad$ Discrepancy analysis . . . . . . . . . . . . . . . . 19

5.2 Performance for medical data . . . . . . . . . . . 19

6 Conclusions . . . . . . . . . . . . . . . 23 


\section{List of Figures}

1 The Rivara's 4 T subdivision approach $\ldots \ldots \ldots$

2 Configurations for triangle subdivision . . . . . . . . 7

3 Configurations for a tetrahedron subdivision . . . . . . . 8

4 Quadrilateral region can be triangulated in two different ways . . . 9

5 Possible tetrahedrizations for Configuration 2a . . . . . . . . 10

6 Eight possible subdivision cases of a triangular prism . . . . . . 11

7 Two possible tetrahedrization for Configuration $3 \mathrm{~b} \ldots \ldots \ldots$

8 Tetrahedrization of Configuration $3 \mathrm{c} \ldots \ldots \ldots$

9 Configuration $4 \mathrm{a} \ldots \ldots \ldots \ldots$

10 Configuration $4 b \ldots \ldots \ldots \ldots \ldots$

11 Possible tetrahedrization of Configuration 5 . . . . . . . . . 14

12 Tetrahedrization of Configuration 6 . . . . . . . . . 15

13 Example of a subdivision process for Configuration $3 \mathrm{~b} \ldots \ldots$

14 Coherence of the subdivision . . . . . . . . . . . . . . 18

15 Perfusion levels of a heart's slice. (a) Original, (b) Original tetrahedral mesh, (c) Threshold=10\%, (d) Threshold $=5 \%$, (e) Scaled discrepancies by a factor of $20 \ldots \ldots 21$

16 Perfusion levels of a heart's slice. (a) Original, (b) Original tetrahedral mesh, (c) Threshold=10\%, (d) Threshold=5\%, (e) Scaled discrepancies by a factor of $15 \ldots \ldots \ldots \ldots$ 


\begin{abstract}
Given a tetrahedral mesh immersed in a voxel model, we present a method to refine the mesh to reduce the discrepancy between interpolated values based on either scheme at arbitrary locations. An advantage of the method presented is that it requires few subdivisions and all decisions are made locally at each tetrahedron. We discuss the algorithm's performance and applications.
\end{abstract}




\section{Introduction}

There are a number of applications (especially in medical imaging, and scientific visualization) that require support for alterations (deformations, cuts and timeevolution) of volume data.

The volume data are often sampled on a regular rectangular grid in 3D space, and stored in a voxel model. Each point on the grid has an associated (scalar or vector) value of a property, that we can think of as a function $f$ whose domain contains the portion of space that we are modelling. To support further computations, or to afford topological flexibility, a simplicial-cells complex would be preferable, and indeed one often immerses into the voxel space such a network of tetrahedra for those purposes. These meshes may be a result of subdividing the voxels into tetrahedra, or may result from tetrahedrizations of a volume extracted from the model (for instance the volume bound by certain iso-surface).

When this kind of mixed models are used, one needs to address the difference in the way voxel models and tetrahedral meshes compute property values away from the sample points. In fact, in the case of voxels the most often used interpolation method to compute values inside a cell is a trilinear interpolation of the sample values known at the vertices of the cell. Analogously, for tetrahedral meshes the most often used method to compute a value inside a tetrahedron is the linear interpolation of the four vertices of that tetrahedron.

It is obvious that even if the initial values assigned to the vertices of the tetrahedra in the immersed mesh are computed from the values in the original volume data, these two different interpolation methods will yield different values of the property at points interior to the tetrahedra.

In this paper we present an algorithm to adaptively subdivide a tetrahedral mesh immersed in a voxel model in order to reduce the discrepancy of these two approximations of the function $f$ below a user-specified tolerance $\varepsilon$. Both the input and output tetrahedral meshes are conformal (i.e. a conforming mesh is one in which two tetrahedra $\mathscr{T}_{i}, \mathscr{T}_{j}, i \neq j$ of the mesh may only intersect at a vertex, along a complete edge or have a common triangular face).

Our algorithm works by locally subdividing tetrahedra where the discrepancy exceeds the tolerance. This paper presents the following contributions:

- Analysis of the discrepancy between the two interpolations within a tetrahedron

- A scheme for locally subdividing tetrahedra with large discrepancies that ensures a conformal resulting mesh and a reduction of the discrepancy within the resulting tetrahedra

- The subdivision scheme aims at minimizing the number of tetrahedra needed to meet the requirement

- The subdivision is designed to yield good quality tetrahedra (not too skinny and elongated) 
We have tested our algorithm over different samples of medical data. Section 5 gives a summary of these tests.

The rest of the paper starts by presenting an overview of previous work in the next section, before presenting our proposed solution in Section 3, where we report on our analysis of the locations of the largest discrepancies and the subdivision patterns (see Section 3.2) used to achieve locality and convergence. Section 4 then discusses some aspects of the implementation of this algorithm, before moving on to the results on our test models, discussed in Section 5, and closing with some conclusions.

\section{Previous Work}

A subdivision scheme can be seen as a procedure to construct a collection of $n$ different meshes $\mathscr{M}=\left\{\mathscr{M}_{1}<\mathscr{M}_{2}<\ldots<\mathscr{M}_{n}\right\}$, such that the mesh $\mathscr{M}_{i+1}$ is obtained from the previous one $\left(\mathscr{M}_{i}\right)$ by a local refinement. At each level, an element is refined if it exceeds a preset error criterion (appropriate for the problem) at that level.

In the context of finite elements, tetrahedral meshes are sometimes subdivided to improve their quality or suitability for the computation at hand. In the related literature a distinction is drawn between two kinds of subdivisions, regular and irregular. Regular subdivision schemes give rise to subtetrahedra that are similar to the tetrahedron being subdivided, whereas irregular schemes do not. At any rate, in order to preserve conformance, when a regular subdivision is performed locally, an irregular scheme must be used to stitch the tetrahedra resulting from the subdivision with their neighbors.

One of the first subdivision schemes that was proposed for two-dimensional triangle meshes, is the red/green or regular/irregular method, by Bank and Sherman [1]. In the red phase, triangles are subdivided into four similar triangles by splitting all three sides at their midpoint. The green phase is subsequently applied to all the neighboring elements that have not been subdivided, but which share exactly one edge with a subdivided triangle. These triangles are split in two joining the midpoint of the edge they share with a subdivided triangle with the opposite vertex. Triangles that have not been subdivided but that share more than one edge with a subdivided triangle are then subdivided using the red strategy, and a subsequent green pass is needed to blend them properly with their neighbors.

Another group of algorithms focuses on the edges, rather than the triangles. This is the case of the work of Rivara [16, 17, 18], in the two-dimensional case. They iteratively apply the longest edge bisection technique. In [16] they use it in its pure form, where the longest edge of each triangle that needs to be subdivided is split at the midpoint, adding an edge to the opposite vertex and resulting in an irregular scheme. In [18] they use instead the 4-Triangles subdivision, where after subdividing a triangle by its longest edge, the midpoint of the longest edge is also joined with two additional edges with the midpoints of the other two edges, 
resulting in a subdivision into 4 triangles (and a regular scheme). Figure 1 shows how this subdivision is performed on a single triangle. Both schemes are supplemented by a pass to insure conformacy, where non-conformant edges are searched, and their coarse neighbors subdivided to achieve conformancy. This needs to be iterated until the mesh is conformant. The authors show numerical evidence that both strategies yield irregular (but conformant) triangulations of good quality.
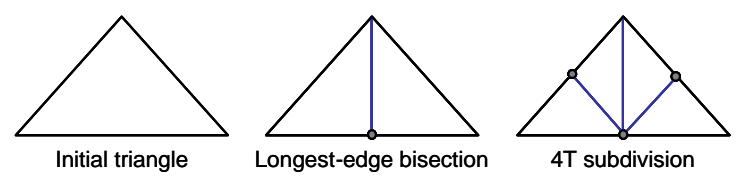

Figure 1: The Rivara's 4T subdivision approach

These strategies have subsequently been extended to the three-dimensional case. Zhang [23], Bey [2], Liu and Joe [10] have extended the strategy of Bank and Sherman [1] to the 3D case. In the regular phase, they subdivide a tetrahedron in eight subtetrahedra. Four subtetrahedra similar to the one being subdivided result from cutting out the four corners of the tetrahedron, at the midpoint of the edges. The remaining four are obtained by subdividing the central octahedron that results from this corner-cutting. The way in which this octahedron is subdivided, and the irregular schemes used to conformally blend the subdivided part of the mesh with its unsubdivided neighbors are the elements that differentiate the proposals listed above. Zhang splits the octahedron by inserting the shortest of its three diagonals (and splitting the volume in four tetrahedra sharing this diagonal in the obvious manner). He shows that this election tends to minimize the degeneracy of the new tetrahedra. Despite being a stable subdivision, for elements with at least one obtuse triangle it can give rise to several different kinds of tetrahedra after successive subdivisions. On the other hand, Bey choose a diagonal implicitly characterized by the order of vertices. In contrast, Liu and Joe map the tetrahedra onto a canonical one, and insert a diagonal joining the midpoints of the two edges mapped onto the longest edges of this canonical one. In relation with the irregular schemes, the patterns presented in $[2,10]$ are incomplete. In [23], irregular scheme are not considered. More recently Greiner and Grosso [7] used a similar regular/irregular scheme, but the interior octahedron is subdivided into six octahedra and eight tetrahedra on demand.

The edge bisection methods discussed for two dimensions have also been extended into 3D. Rivara and Levin [19] extended first the pure bisection method by simply bisecting tetrahedra by splitting the longest edge and joining the split point with the opposite vertices of the adjacent faces. Liu and Joe [9] later showed by numerical experiments that this may lead to the bisection of many tetrahedra, which not only increases the cost of the subdivision, but more importantly may severely impact the finite elements computation. Instead, they propose to map the tetrahedron onto a canonical one, and show how to use this to subdivide the tetrahedron 
into eight similar pieces, in a three steps process.

Plaza and Carey $[12,13]$ have extended to three dimensions the 4-Triangle algorithm. After selecting the set of tetrahedra that needs to be refined, they insert new vertices at the midpoint of each edge. Then they explore the neighbors to verify it conformity. If the tetrahedron is non conforming, insert new vertices at the midpoint of the longest edge of each non conforming face and add a new vertex to the midpoint of the longest edge of the tetrahedra. From this points, it makes the subdivision of each face using the 4-Triangle algorithm, obtaining the skeleton. Once the set of faces have been consistently triangulated in this way, they complete the subdivision of the interior of the tetrahedra based on a set of 51 different precomputed patterns. More recently, they do a new proposal denominated "8-Tetrahedra longest edge partition" $[15,14]$.

In Computer Graphics applications, refinement algorithms used for multiresolution purposes are intended to allow the acceleration of the visualization and interaction processes. They subdivide the volume data in tetrahedral meshes with different levels of detail (LoD). These algorithms use both subdivision and fusion techniques [4, 24]. In Danovaro et al.'s work [4], two multiresolution strategies with different refinement rules are compared. Regular meshes are refined by using the bisection rule, and unstructured meshes are refined with vertex split rule. On the other hand, Zhou et al. [24] propose a hierarchy of tetrahedra obtained by a recursive subdivision of the volume. Three subdivision rules and an error saturation strategy are defined for the multiresolution.

On the other hand, adaptive subdivision of triangle meshes for deformable models is presented by Ruprecht et al. [21]. They apply adaptive subdivision for deformable models used in volumetric data matching or volumetric morphing. The subdivision is carried out when the distance between the edge midpoints in real space and the same points in the deformed space is greater than certain $\varepsilon$. They use this adaptive subdivision strategy in [22], to subdivide tetrahedral meshes. Their subdivision scheme is similar to the one we propose, although we solve differently the cases with additional degrees of freedom.

Yet another field of application where a need for these subdivisions arises is surgery simulation. Here one wants to simulate cuts into volumetric models based on tetrahedral meshes. Cuts are simulated by subdividing the tetrahedra intersected with the virtual scalpel. These techniques differ slightly from the previous methods because the subdivision points are given by the user interaction, and have to be duplicated in order to separate the mesh along the cutting line $[6,3,5,11]$.

Neither of the previous subdivision techniques takes into account in the subdivision process the volumetric information contained in the interior of the tetrahedra. In our application we focus on the extraction of a tetrahedral mesh from the volume data, and are therefore concerned with how well does the extracted mesh agree with the model in terms of the estimates of the property of interest in its interior points. Thus, we define the subdivision rules according to the interior information of the volumetric data. We are not aware of previous results in the literature that address this problem in these terms. 


\section{Our proposal}

We are interested in the case where we have a hybrid model, consisting on volume data in the form of a voxelization, and a tetrahedrization of a portion of the same volume, where the vertices of the tetrahedrization are in arbitrary positions within the volume. For instance, these tetrahedrization may come from the computation of an active-contour-like triangle mesh delimiting a portion of interest of the volume, followed by a subdivision of that volume compatible with the triangulation of the boundary.

Notice that in this general setup a tetrahedron may span several voxels, or several tetrahedra may be completely contained inside a voxel. The discrepancies that we want to minimize may therefore come strictly from the difference in interpolation (in the second case), or may originate in rapidly varying volume data (in the first case).

The next subsection discusses the nature of these discrepancies in a formal way. However, we have not reached a useful closed-form solution for the optimal way to subdivide tetrahedra, therefore we have resorted to experiments, which are discussed in Section 5. From these numerical experiments, we have seen that most of the time the point of maximum discrepancy happens near the midpoint of a face or edge. When the maximum occurs at the midpoint of a face, a similar value of the discrepancy appears near the midpoint of at least one of its edges. For this reason, and in the interest of speed, we chose to analyze only the edge midpoints of the given tetrahedra, splitting an edge at its midpoint if the discrepancy there exceeds a threshold. This has the interesting property of providing a completely local test. In the subsection 3.2 we discuss the possible configurations and present a scheme for subdividing the tetrahedra that minimizes the number of resulting tetrahedra, and is based exclusively on these local computations (therefore there is no need to propagate subdivisions, as decisions in neighboring tetrahedra are guaranteed to be consistent and yield a valid tetrahedrization). When an edge is split at its midpoint $Q$ (also called here a splitting point), $Q$ is assigned a new property value equal to realValue $(Q)$. We find that the discrepancies in the new tetrahedra decrease, and the scheme quickly converges.

\subsection{On the computation of discrepancies within a tetrahedron}

Let $\mathscr{M}$ be a tetrahedral mesh immersed in a voxel model $\mathscr{V}$. Let us further assume that we assign to each vertex $v \in \mathscr{M}$ a property value obtained by trilinear interpolation of the corners of the cell that contains $v$ in the voxel model. A tetrahedron $\mathscr{T} \in \mathscr{M}$ is called a good predictor if for any point $P \in \mathscr{T}$ the discrepancy between the property value computed at $P$ from the voxel model (by trilinear interpolation of the vertices of the cell that contains it) and from the tetrahedral mesh (by linear interpolation of the property values at the vertices of $\mathscr{T}$ ) is below a user-specified threshold $\varepsilon$.

That is, if $P$ has coordinates $(x, y, z)$ within its cell (i.e. $x, y, z \in[0,1])$, then we 
define

$$
\begin{aligned}
\text { realValue }(P)= & (1-x)(1-y)(1-z) I_{000}+(x)(1-y)(1-z) I_{100}+ \\
& (1-x)(y)(1-z) I_{010}+(x)(y)(1-z) I_{110}+ \\
& (1-x)(1-y)(z) I_{001}+(x)(1-y)(z) I_{101}+ \\
& (1-x)(y)(z) I_{011}+(x)(y)(z) I_{111}
\end{aligned}
$$

where the $I_{i j k}$, denote the values at the corresponding corners of the cell.

Moreover, let $b_{i}, i=0 \ldots 3$ be the barycentric coordinates of the point $P$ with respect to its tetrahedron $\mathscr{T}$ (which satisfy $0 \leq b_{i} \leq 1 \forall i$ and $b_{0}+b_{1}+b_{2}+b_{3}=1$ ), and let $I_{v i}$ be the values assigned to the vertices of $\mathscr{T}$. Then we define

$$
\operatorname{aproxValue}(P)=b_{0} I_{v 0}+b_{1} I_{v 1}+b_{2} I_{v 2}+b_{3} I_{v 3} .
$$

The condition that $\mathscr{T}$ is a good predictor can then be written as

$$
\forall P \in \mathscr{T}: \frac{\mid \text { realValue }(P)-\text { aproxValue }(P) \mid}{\text { normCoeff }} \leq \varepsilon
$$

where normCoeff is a normalization coefficient so that all values are in $[0,1]$ (and discrepancies measure relative error).

We shall also denote the discrepancy at a point $P$ by $\operatorname{error}(P)=\mid$ realValue aproxValue|/normCoeff. If error $>\varepsilon$ the tetrahedron is not a good predictor and must be subdivided. Choosing $\varepsilon$ is relatively straightforward for the user, as it represents relative error. A value of 0.1 , for example, indicates that errors below $10 \%$ are acceptable.

\subsection{Subdivision scheme}

We need to consider the different configurations of edges of a tetrahedron that need to be split. We would like to achieve a scheme that does not impose a subdivision of an edge that was not marked for splitting to begin with. This is both related to minimizing the number of resulting tetrahedra and to making the scheme local (an edge needs to be split based on an intrinsic property, and not on the configuration of its neighbors).

Let us first linger for a moment in the simpler two-dimensional case, where triangles are subdivided by breaking their edges. Since a triangle has three edges, there are $2^{3}=8$ different configurations. The extreme configurations, in which no edge is subdivided and all three edges are subdivided, only happen once each one. The cases where only one edge is subdivided and where two edges are subdivided occur three times each. The 8 configurations are thus reduced to 4 due to symmetries, shown in Figure 2. Notice that the quadrilateral region in case 2 can be triangulated in two different ways. Some authors solve the ambiguity by using the memory addresses of the different vertices. Instead, we choose to add the shortest of the two diagonals of the quadrilateral region to split it into two triangles. This yields better shaped tetrahedra (see [16]). 

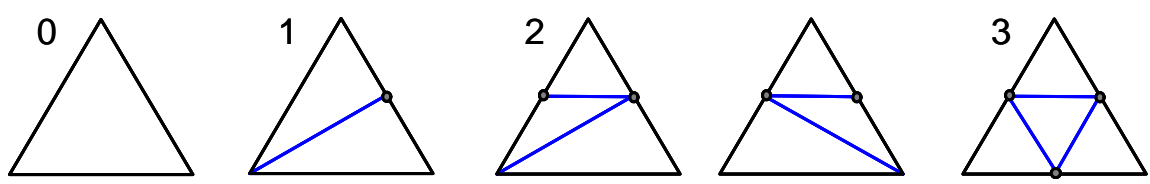

Figure 2: Configurations for triangle subdivision

We can therefore construct a LUT with eight entries (indexed by the vertex classification), sorting the different configurations into one of the four cases depicted above. However, when we reach configuration number two, we need to also compute the shortest diagonal of the quadrilateral portion in order to decide the sub-case applicable. This yields a total of 11 possible triangulations:

$$
\begin{aligned}
\text { TotalPatterns } & =\sum_{i=0}^{3} 2^{N Q R_{i}} * N E_{i} \\
& =2^{0} * 1+2^{0} * 3+2^{1} * 3+2^{0} * 1 \\
& =11
\end{aligned}
$$

Here $N Q R$ is the number of quadrilateral regions ( 0 or 1$)$ and $N E$ is the number of distinct rotations of the configuration.

Let us now consider the three-dimensional case. Our tetrahedron subdivision scheme will produce exactly these subdivisions on the faces of the tetrahedra it subdivides. Since neighboring tetrahedra share a face, and they both get subdivided in a way that is consistent with that face, the result is automatically conformal.

Tetrahedra have 6 edges, so there are $2^{6}=64$ possible edge refinement patterns. Removing cases that differ by a symmetry or a rigid motion, the 64 cases are reduced to 11 different configurations, shown in Figure 3. As in the two-dimensional case, we compute the length of both diagonals of the quadrilateral region, and then split the quadrilateral along the shortest diagonal. This yields better triangles, and therefore tetrahedra with better quality. If both diagonals have equal length, the one containing the vertex with smaller id on the mesh data structure is selected. This allows us to guarantee that these faces, when shared by two tetrahedra, are triangulated without ambiguity, as shown in Figure 4. Let us now examine these configurations in detail.

Configuration 0 in Figure 3 corresponds to the trivial case where no edge needs refining, and the tetrahedron is not subdivided further. Almost as simple is the case of Configuration 1, where a single edge needs subdividing. Two subtetrahedra are obtained by joining the new midpoint with the opposite vertices. Notice that the solution adopted influences only faces sharing the edge with excessive error, which is relevant to ensure that the resulting scheme is local, as neighbors will automatically make consistent decisions.

There are two distinct cases where two different edges need to be subdivided: 

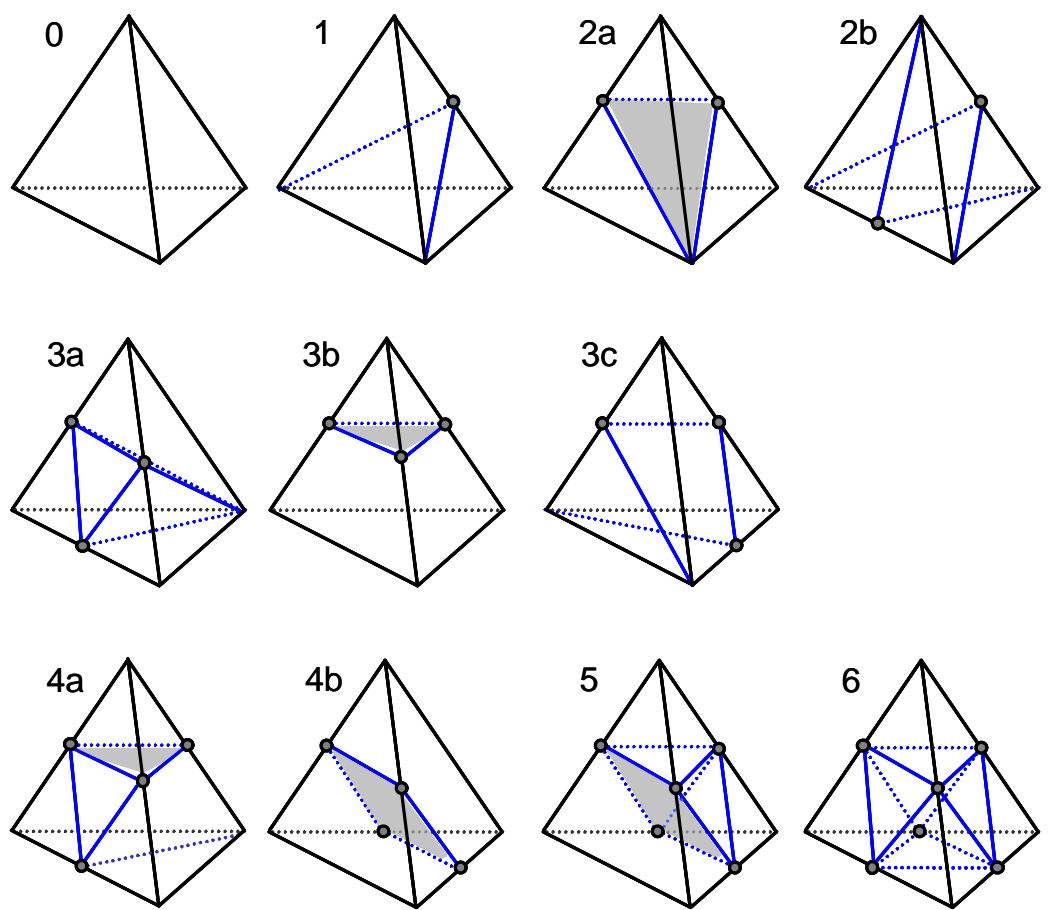

Figure 3: Configurations for a tetrahedron subdivision

Configuration 2a: The two edges belong to the same face. This case yields a subdivision into three tetrahedra. Figure 5 shows the two different tetrahedrizations that may result from this step.

Configuration 2b: The two edges to divide are opposite edges of the tetrahedron. This case can be solved by applying the solution for configuration 1 twice in succession. In this way the tetrahedron is split in four sub-tetrahedra, as shown in Figure 3

In the case where three edges exhibit errors above the chosen threshold, three different configurations may arise:

Configuration 3a: the three edges needing subdivision belong to the same face. The face is divided in four triangles with new edges connecting the error points. Each new triangle is joined with the opposite vertex and the tetrahedron is thus subdivided into four sub-tetrahedra.

Configuration 3b: The three edges have a common vertex. All three triangular faces sharing that common vertex have two edges with errors above the threshold value. A sub-tetrahedron is formed by that vertex and the three midpoints of the converging edges. The remaining prism can be subdivided in eight different ways, depending on the lengths of the diagonals of each 


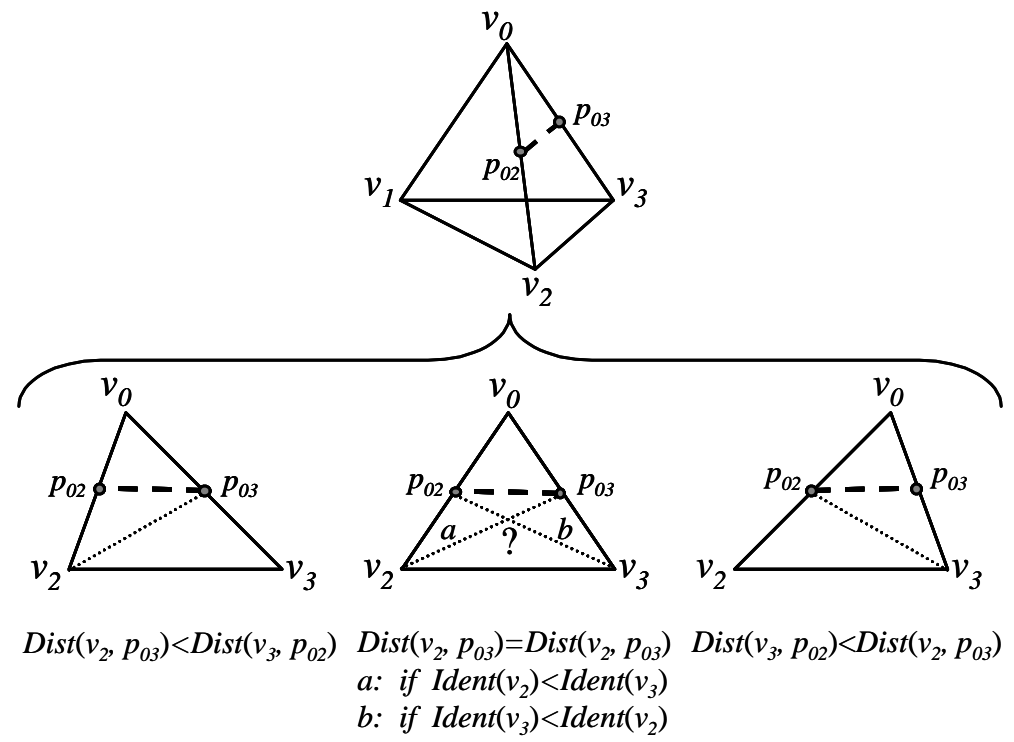

Figure 4: Quadrilateral region can be triangulated in two different ways

quadrilateral face of the prism (see Figure 6). An example of two base resulting cases are shown in Figure 7 at right. The first one (the top right sub-figure) arises when two of the shorter diagonals converge at a splitting point ( $p_{03}$ in the figure). In that case the prism is subdivided into three tetrahedra (i.e. in the figure, the tetrahedra with vertices $\left(v_{2}, p_{01}, p_{02}, p_{03}\right)$, $\left(v_{1}, v_{2}, p_{01}, p_{03}\right)$, and $\left.\left(v_{1}, v_{2}, v_{3}, p_{03}\right)\right)$.

The second possibility (down right sub-figure) occurs when no two of the shorter diagonals of the three quadrilaterals begin/finish at the same point $(x=\emptyset$ in the Algorithm 1). In this case, an untetrahedralizable region known as a Schönhardt prism is formed. This prism cannot be broken up into tetrahedra whose vertices are vertices of the prism unless the triangulation of one of the three quadrilateral facets is changed by doing an edge flip [20]. We want our tetrahedron subdivision to depend only on local information, so we cannot afford this edge flip (which would go unnoticed to the neighbor tetrahedron). Instead, we add a Steiner point inside the prism to guarantee coherence of the subdivision. Then, each triangular face is joined with the Steiner point. As a result, the prism is subdivided into eight tetrahedra. A pseudo-algorithm to resolve this case is shown in Algorithm 1.

Configuration 3c: Two of the three edges where the error exceeds the threshold are opposed. The third edge shares two different facets of the tetrahedron, one with each of these two opposed edges, as shown in Figure 8 at left. We break up these tetrahedra in five sub-tetrahedra as follows: first, consider 


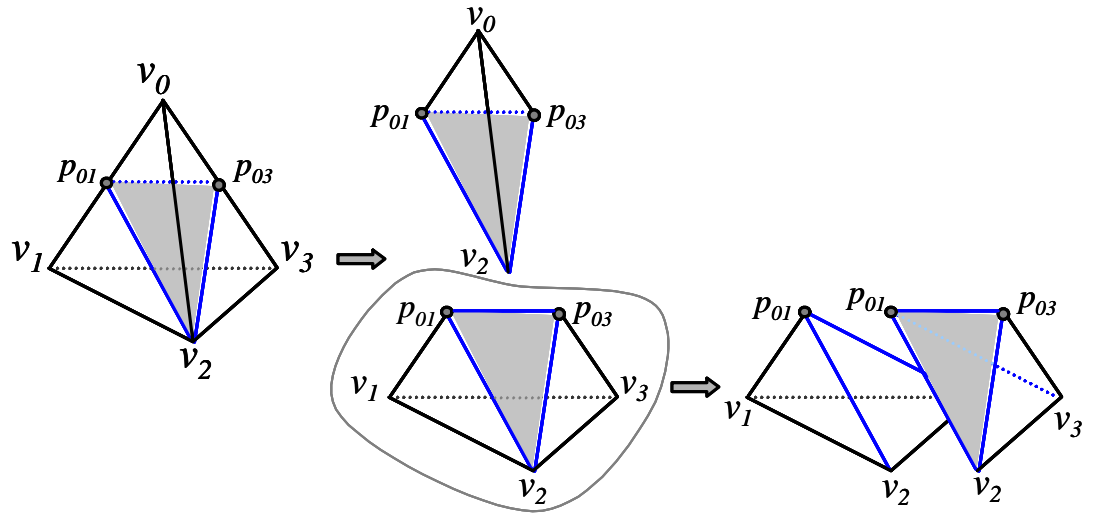

Figure 5: Possible tetrahedrizations for Configuration 2a

the facets of the tetrahedron that have only one splitting point $\left(v_{0}, v_{1}, v_{2}\right.$ and $v_{1}, v_{2}, v_{3}$ in the figure), and split these faces adding an edge from the splitting point to the opposite vertex. We thus obtain the first sub-tetrahedron, whose edges are these four points $\left(v_{1}, v_{2}, p_{01}\right.$ and $p_{23}$ in the figure). What remains is the union of two pyramids with apices at these two splitting points $\left(p_{01}\right.$ and $\left.p_{23}\right)$, and with quadrangular bases $\left(v_{0}, v_{2}, p_{23}, p_{03}\right.$ and $v_{3}, v_{1}, p_{01}, p_{03}$ respectively). These two pyramids share a triangular face whose vertices are the three splitting points as shown on the right of Figure 8. Each of these pyramids is broken up into two tetrahedra by splitting its base along the shortest diagonal.

There are two different cases where four edges need to be subdivided:

Configuration 4a: In this case three of the four edges with split points belong to the same face of the tetrahedron. The fourth one necessarily shares one vertex with that face. This configuration is depicted on the left side of Figure 9. It is sort of a mixture of the configurations $3 a$ and $3 b$ discussed above. We subdivide this configuration by forming two tetrahedra sharing the triangle $p_{01}, p_{02}, p_{03}$ in the figure (i.e. the triangle formed by the split points on three converging edges). These tetrahedra are $\left(v_{0}, p_{01}, p_{02}, p_{03}\right)$ and $\left(p_{01}, p_{02}, p_{03}, p_{12}\right)$ in Figure 9 . The remaining volume within the tetrahedron consists again of two pyramids with quadrangular bases separated by the triangle $p_{12}, p_{03}, v_{3}$. These pyramids are finally subdivided into two tetrahedra each one using the shortest diagonal criterion. The right side of Figure 9 shows a blow-up of these two pyramids.

Configuration 4b: Each face of the tetrahedron has exactly two edges with split points. The split points are the vertices of a quadrangle that splits the tetrahedron into two similar prisms (see Figure 10). 

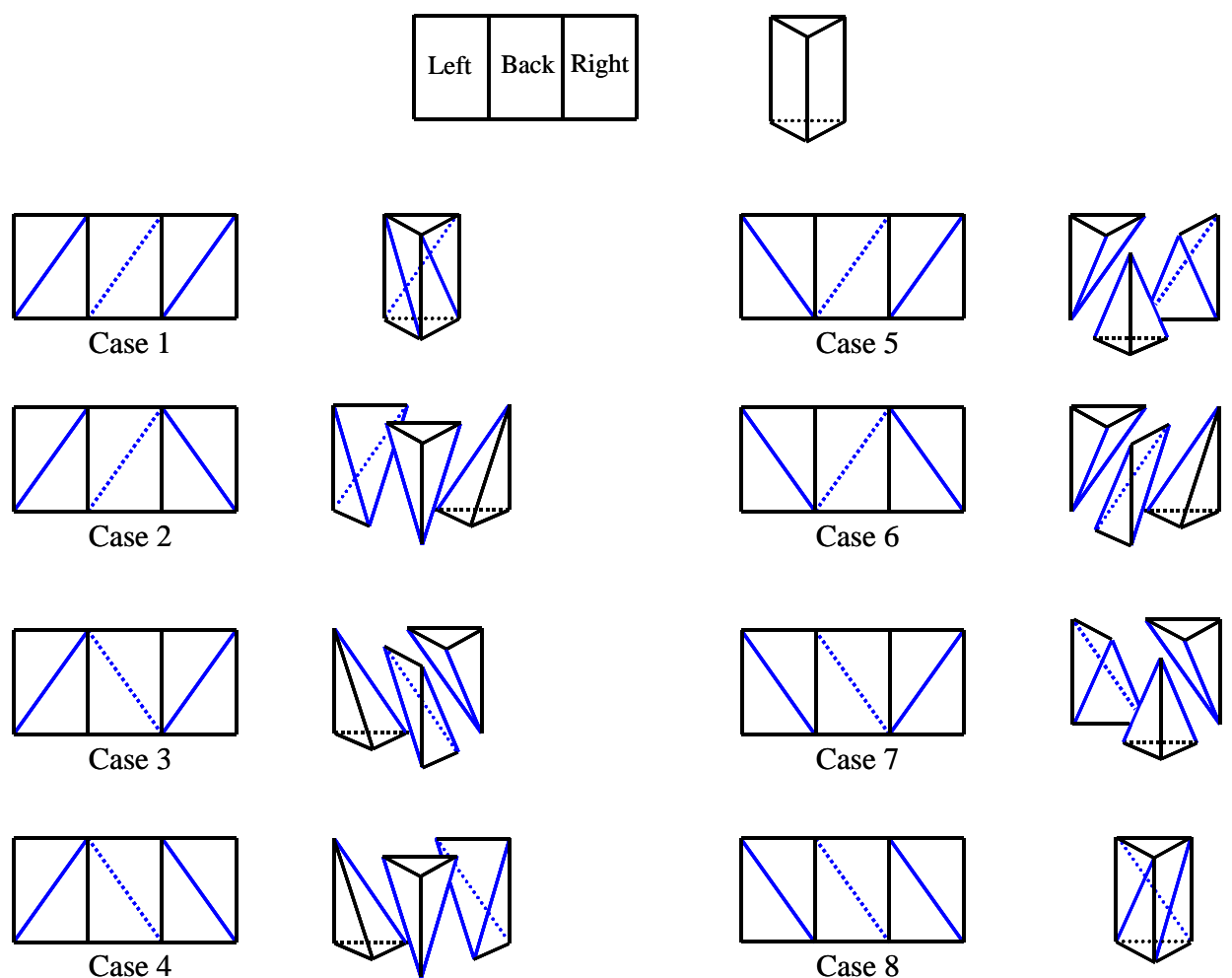

Figure 6: Eight possible subdivision cases of a triangular prism

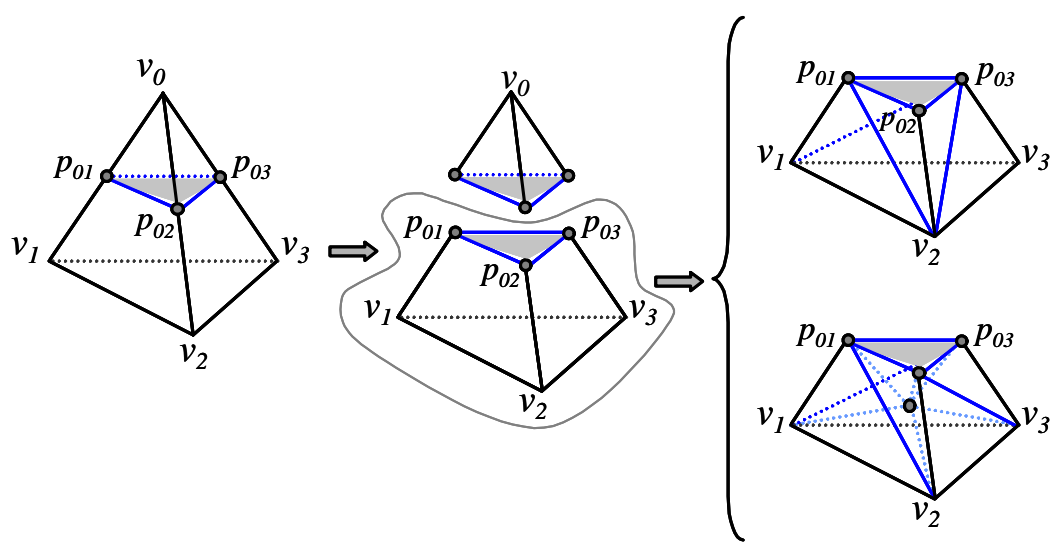

Figure 7: Two possible tetrahedrization for Configuration $3 b$ 


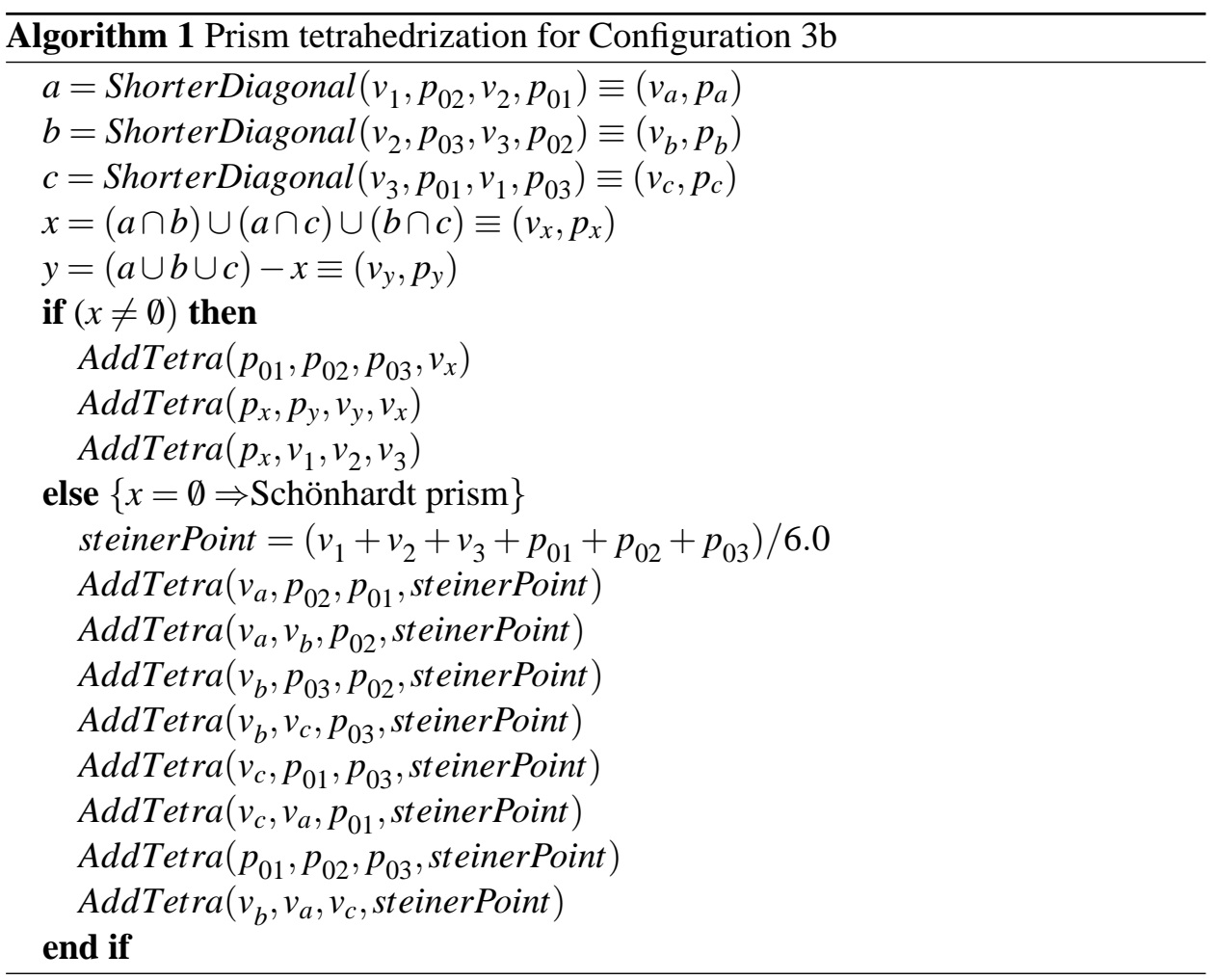

To tetrahedrize each of the two prisms, we consider the shortest diagonal of each of the quadrangular faces contained in the boundary of the tetrahedron. For the first prism to be tetrahedrized, two cases are possible, depending on whether its two shorter diagonals of the exterior quadrangles have a point in common or not. If they do (Figure 10, top right) then we are still free to split the central quadrangle, so we look at the tetrahedrization of the second prism, in order to minimize the probability of producing Schönhardt prisms. If they do not (Figure 10, down right) then the interior quadrangle is split joining the endpoints of these diagonals. The second prism will inherit the choice of diagonal for the interior quadrangle. If it forms a Schönhardt prism, we have no degree of freedom left, and we must introduce a Steiner point to tetrahedrize it.

Configuration 5: This is the case where there are five split points on the edges of the tetrahedron (see Figure 11). The figure shows how the tetrahedron is naturally split into a prism like one of those in Configuration $4 \mathrm{~b}$, a pyramid with quadrangular base, and two tetrahedra. The prism is tetrahedrized like the first prism in Configuration $4 \mathrm{~b}$, determining the diagonal to use in the interior quadrangle shaded in grey, which determines the splitting of the pyramid and hence the whole tetrahedrization.

Configuration 6: All six edges of the tetrahedron contain a split point. Joining 


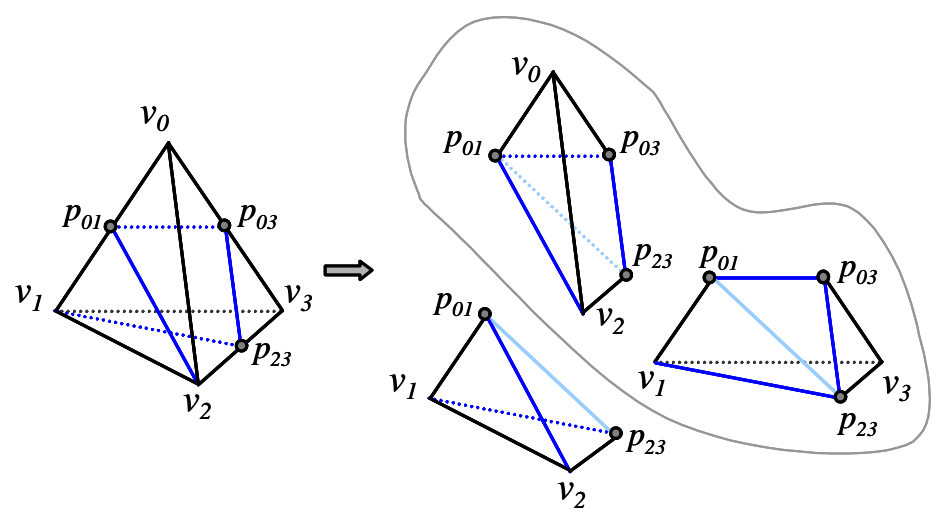

Figure 8: Tetrahedrization of Configuration 3c

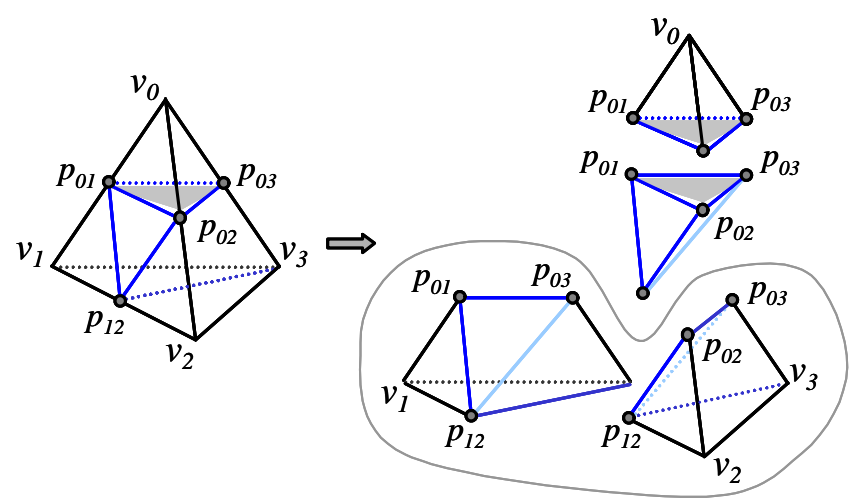

Figure 9: Configuration 4a

the split points on the edges that converge to each vertex of the tetrahedron, we obtain four small corner tetrahedra, and a central octahedron. The octahedron is tetrahedrized inserting the shortest of the three internal diagonals that join split points on opposed edges, and thus dividing it into four more tetrahedra (see Figure 12).

An analysis of the different cases just discussed will convince the reader that when a triangular facet is shared by two tetrahedra, the splitting on both neighbors will be consistent at the facet: every facet with just one splitting point will have been broken up in two triangles by joining that splitting point with the vertex opposed to it; every facet with two splitting points will have been broken up into three triangles, by adding an edge joining the two splitting points, plus the shortest of the two diagonals of the remaining quadrangle; and every facet with a splitting point on 


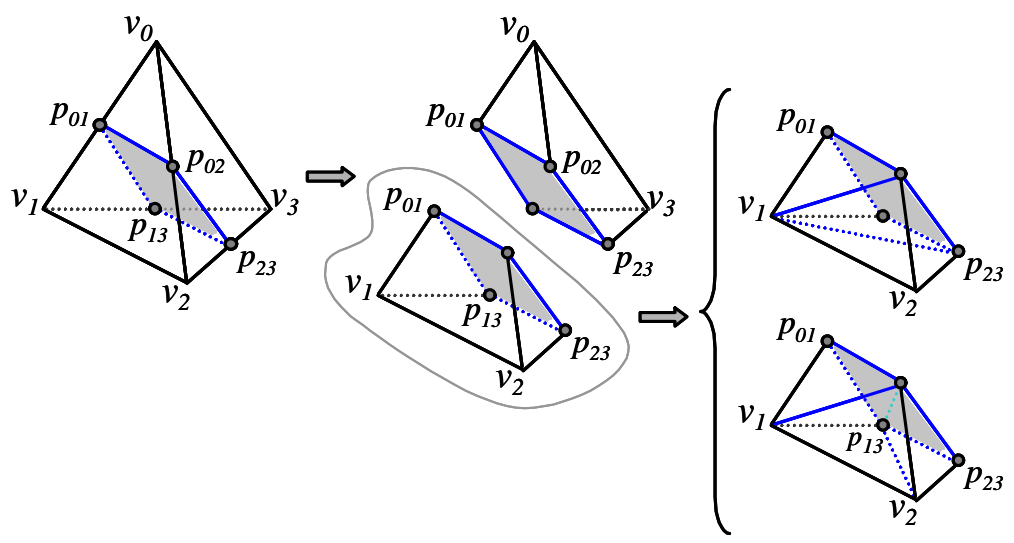

Figure 10: Configuration 4b

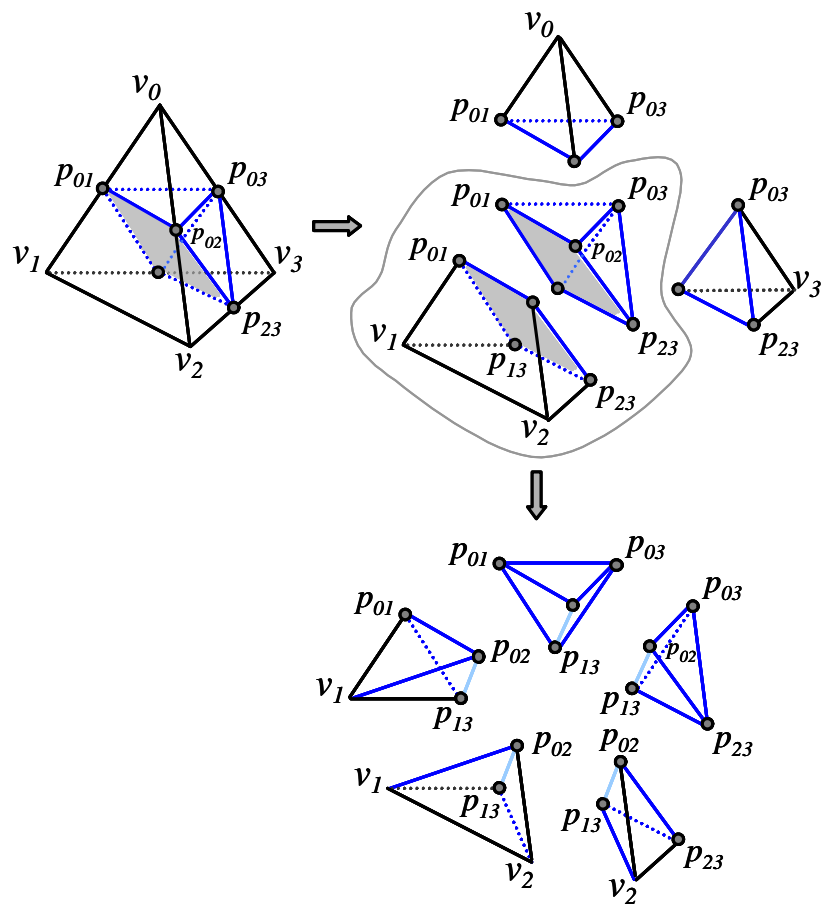

Figure 11: Possible tetrahedrization of Configuration 5 


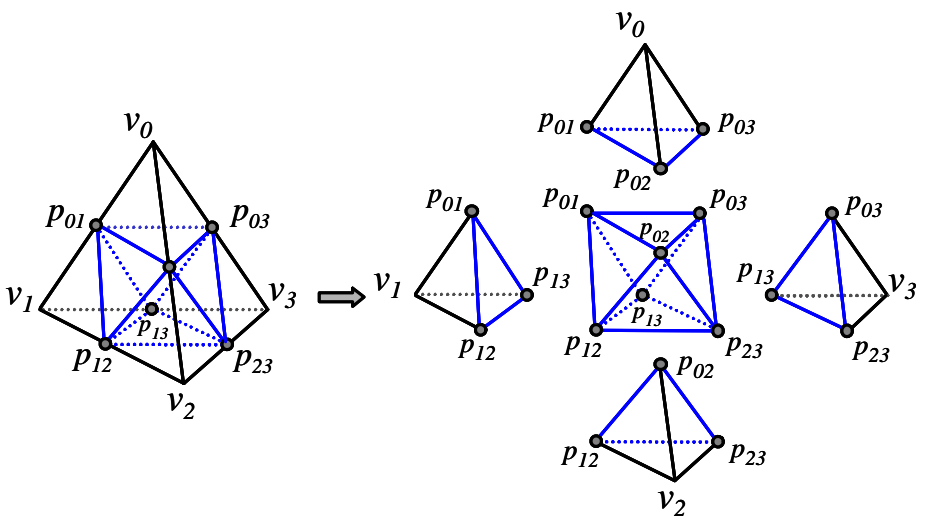

Figure 12: Tetrahedrization of Configuration 6

each edge will have been broken up into four triangles by adding the three edges defined by the three splitting points. Therefore this subdivision scheme allows for completely local decisions and automatically produces conformal tetrahedrizations. Note that the discrepancies along edges will coincide regardless of which tetrahedron is used to compute them, so indeed all neighboring tetrahedra see the same configuration and therefore consistent decisions are made on all neighbors.

\section{Implementation details}

Our implementation uses a lookup table indexed by the 64 possible arrangements of splitting points on a tetrahedron. Each entry contains a specific case and the order in which the vertices must be processed. Table 1 summarizes the possible cases:

Here the column labeled "Case" lists the configuration number as in the discussion of the previous section and in Figure 3 above. The second column shows the number of sub-tetrahedra into which the tetrahedron being considered is split. In cases $3 b$ and $4 b$, where a Shönhardt prism may arise, include in parenthesis the number of tetrahedra needed in that case. The third column indicates the number of entries in the lookup table (NE) corresponding to each base case (taking symmetries and rigid motions into account), and adds up to the 64 relevant cases mentioned above. The last column, finally, indicates the number of different tetrahedrizations (NDT) that can arise in each base case because of the different possible subdivisions of the quadrangular facets. In cases $4 \mathrm{~b}$ and 5, NDT is not a power of two because the subdivision of the interior quadrilateral is not always free.

The proposed 11 subdivision patterns yield thus 269 different tetrahedrizations, all based on local criteria and guaranteeing the conformity of the result.

Our algorithm proceeds as follows. Initially, we compute values for each ver- 


\begin{tabular}{|c||c|c|c|}
\hline Case & $\|\mathscr{T}\|$ & NE & NDT \\
\hline \hline 0 & 1 & 1 & 1 \\
1 & 2 & 6 & 1 \\
$2 \mathrm{a}$ & 3 & 12 & 2 \\
$2 \mathrm{~b}$ & 4 & 3 & 1 \\
$3 \mathrm{a}$ & 4 & 4 & 1 \\
$3 \mathrm{~b}$ & $4(9)$ & 4 & 8 \\
$3 \mathrm{c}$ & 5 & 12 & 4 \\
$4 \mathrm{a}$ & 6 & 12 & 4 \\
$4 \mathrm{~b}$ & $6(11)$ & 3 & 22 \\
5 & 7 & 6 & 6 \\
6 & 8 & 1 & 1 \\
\hline
\end{tabular}

Table 1: Summary of the lookup table

tex of the given tetrahedrization from the voxel data, using trilinear interpolation. Then the error at the midpoint of each edge is computed and compared with the threshold. For each tetrahedron, the collection of edges exceeding the threshold determines an index into the lookup table. If a subdivision is required (all cases except configuration 0 ), the tetrahedron is removed, and replaced by the sub-tetrahedra described above (in those configurations which involve quadrangular sub-facets, the shortest diagonals are computed to determine the appropriate subdivision). The lookup table store five different values. The first value represents the subdivision case. The remaining four values are the order in which the vertices must be sorted so as to match the standard configuration for that case. This maps each possible rotation or symmetry onto a canonical position so the algorithm not worry about these transformations. A pseudo-code description of the algorithm is shown in Algorithm. 2.

Figure 13 shows an example of the program at work with a tetrahedron with configuration $3 \mathrm{~b}$. The example shows how the code computes the resulting subdivision without computing the transformation from the given setting to the standard configuration. Instead, intrinsic properties are used to find the vertices of the new tetrahedra, greatly simplifying the code.

Let us again stress that the subdivision defined gives consistent subdivisions to neighboring tetrahedra, based on the analysis of the common facet alone (see Figure 14). This is the reason why we have chosen to add an extra vertex in configurations $3 b$ and $4 b$ to tetrahedrize the Schönhardt prism, instead of trying to avoid it, despite the greater number of tetrahedra required in this (certainly infrequent) case. 


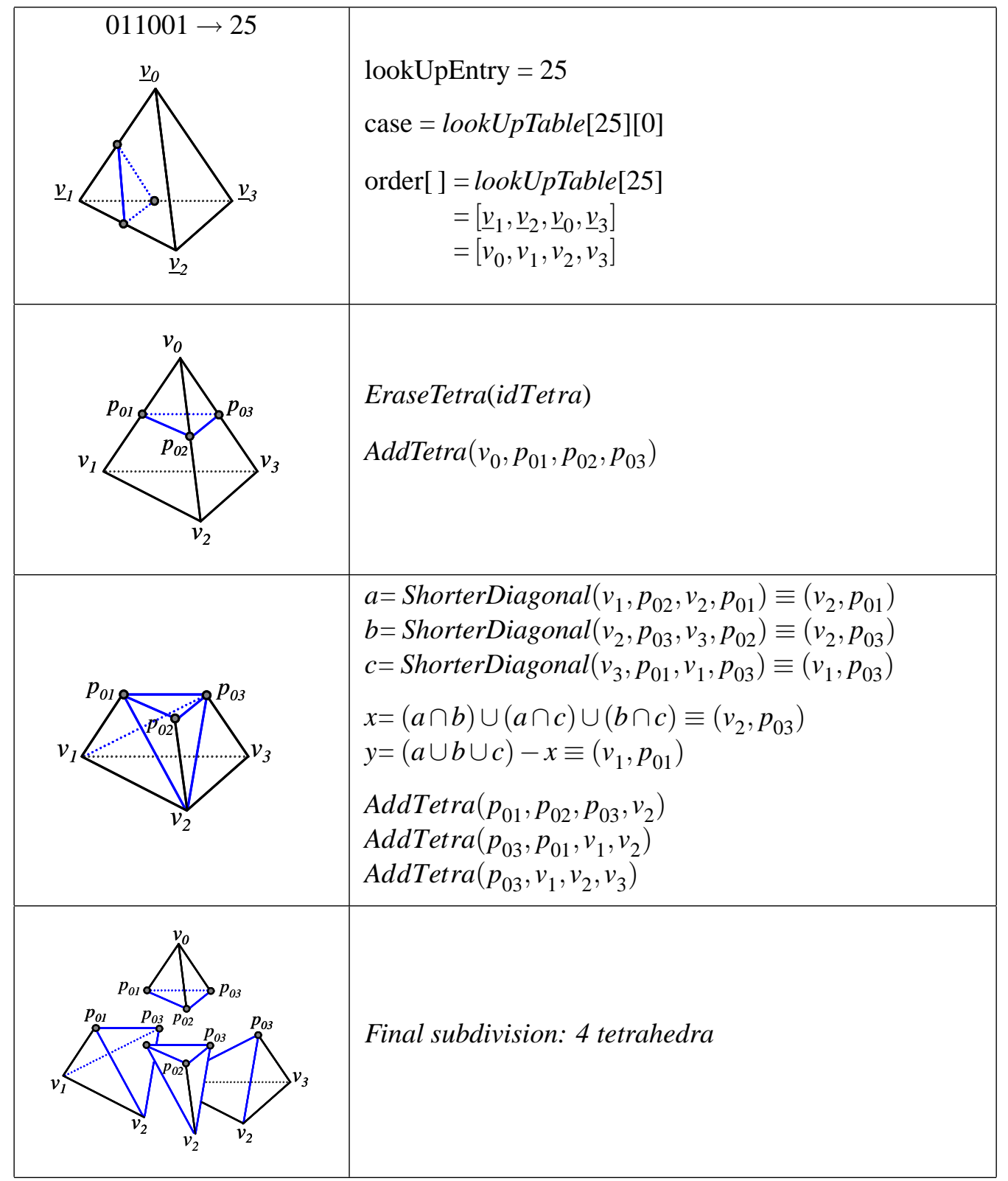

Figure 13: Example of a subdivision process for Configuration $3 \mathrm{~b}$ 

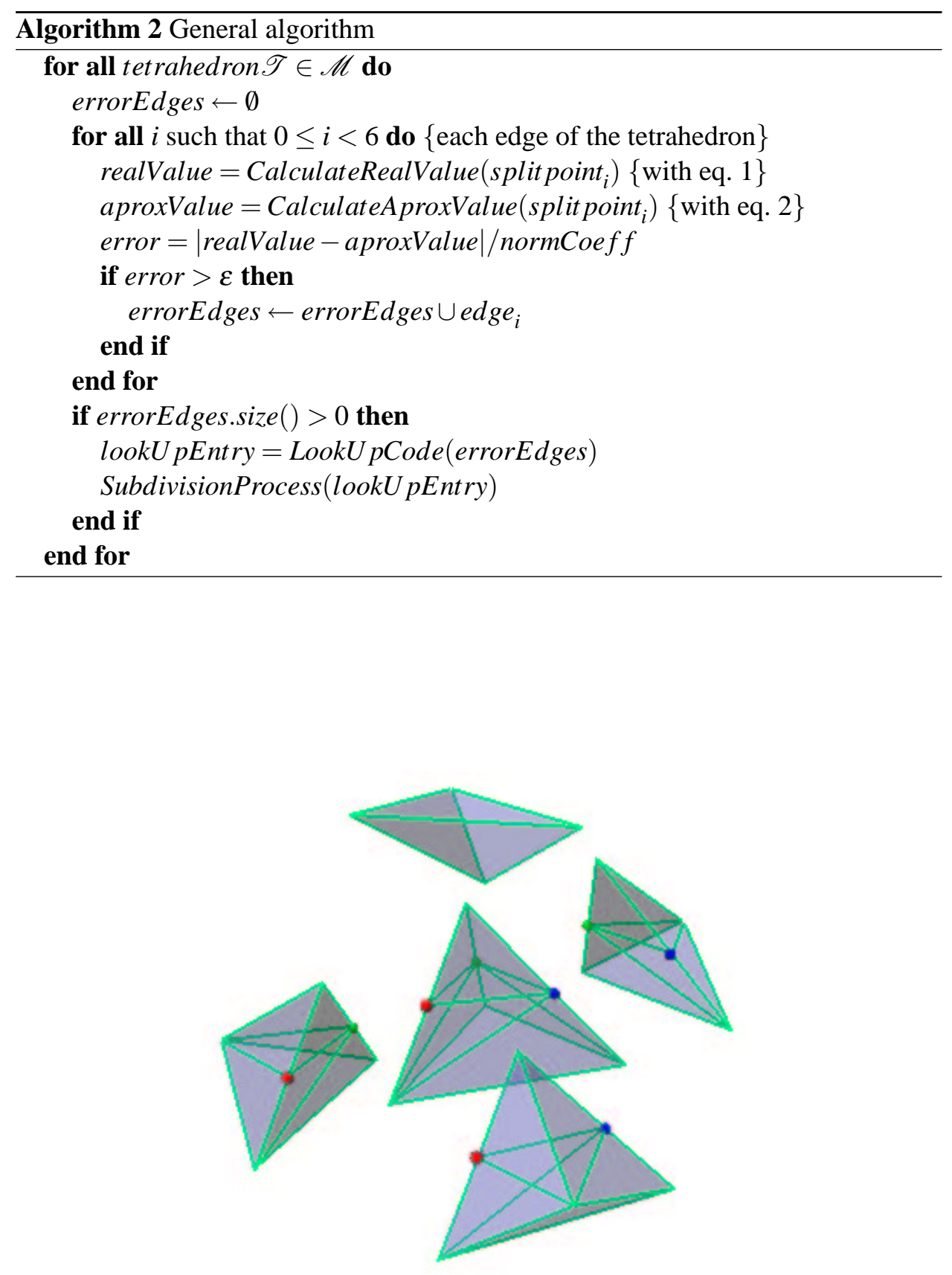

Figure 14: Coherence of the subdivision 


\section{Experimental Results}

We have performed two different kinds of experiments. The first one was addressed at studying the distribution of the discrepancy inside tetrahedra, and the second at measuring the performance of our subdivision algorithm on real testcases.

\subsection{Discrepancy analysis}

To determine the behaviour of the discrepancy within a tetrahedron, we computed the value of the left-hand side of inequality (3) at regularly spaced points (those with barycentric coordinates of the form $\left.\left(\frac{i}{n}, \frac{j}{n}, \frac{n-i-j}{n}\right), i, j \in\{0, \ldots, n\}\right)$.

First we tested regular subdivisions of voxels - into five tetrahedra each - to measure the part of the discrepancy due to the difference in interpolation schemes. We estimated the error in each tetrahedron by sampling 165 equally spaced points ( $n=8$ in the formula above).

In this experiment, $93 \%$ of the time the maximum discrepancy happened at the midpoint of one of the edges. In the remaining cases the maximum occurred at a face of the tetrahedron (i.e. one of the barycentric coordinates was zero). In these cases, however, at least one of the edges of the face exhibiting the maximum discrepancy had a discrepancy of the same order.

To assess the contribution to the discrepancy coming from the higher sampling rate of the voxels, we run further tests with coarse tetrahedrizations of volume models. We set a threshold for the relative error such that discrepancies below that threshold were ignored. The results obviously presented more variability. However, for a threshold of 0.001 , we still found that in over $73 \%$ of the cases (again computed taking $n=8$ ), the maximum discrepancy occured large at the midpoint of some edge or in a point near to this one. Increasing the threshold to 0.05 increased the number of hits to $85 \%$.

Based on these results we concluded that a more precise estimation of the position of maximum discrepancy would probably not yield substantial performance benefits, and decided to proceed with a straightforward test based on comparing the errors at the midpoints of the tetrahedra to decide wich edges to split. This choice seems to be confirmed by experiments on medical datasets that are the subject of the next subsection.

\subsection{Performance for medical data}

We have tested our algorithm on several medical datasets. The heart models were obtained with SPECT and the liver was obtained with MRI. The MRI model has been undersampled to a low resolution. Table 2 shows the resolutions and voxel sizes for three of these models.

For the heart data we also had reconstructions of the inner and outer surfaces of the ventricle, and proceeded to build a tetrahedrization between them, as presented in [20]. For the liver model, a tetrahedrization of the voxels was performed instead. 


\begin{tabular}{|c||c|c|}
\hline & Resolution & voxel size (in mm.) \\
\hline \hline heart1 & $64 \times 64 \times 24$ & $2.87 \times 2.87 \times 5.74$ \\
heart2 & $32 \times 32 \times 11$ & $10.776 \times 10.776 \times 10.776$ \\
liver & $16 \times 16 \times 16$ & $24.5624 \times 24.5624 \times 3.19998$ \\
\hline
\end{tabular}

Table 2: Medical data sets used

\begin{tabular}{|c||ccc|ccc|ccc|}
\hline Case & & heart1 & \multicolumn{3}{|c|}{ heart2 } & \multicolumn{3}{c|}{ liver } \\
& Iter. 1 & Iter. 2 & Iter. 3 & Iter. 1 & Iter. 2 & Iter. 3 & Iter. 1 & Iter.2 & Iter. 3 \\
\hline \hline 0 & 231 & 2453 & 4452 & 244 & 2744 & 3990 & 7307 & 17149 & 24739 \\
1 & 110 & 348 & 31 & 117 & 327 & 33 & 984 & 1793 & 566 \\
2a & 130 & 212 & 12 & 145 & 148 & 20 & 638 & 1354 & 246 \\
$2 \mathrm{~b}$ & 2 & 9 & 1 & 3 & 0 & 0 & 40 & 157 & 1 \\
$3 \mathrm{a}$ & 1 & 0 & 0 & 3 & 0 & 0 & 15 & 9 & 0 \\
$3 \mathrm{~b}$ & 325 & 114 & 0 & 280 & 28 & 0 & 1355 & 11 & 0 \\
$3 \mathrm{c}$ & 36 & 21 & 0 & 29 & 12 & 0 & 120 & 7 & 0 \\
$4 \mathrm{a}$ & 13 & 0 & 0 & 15 & 0 & 0 & 89 & 1 & 0 \\
$4 \mathrm{~b}$ & 97 & 19 & 0 & 143 & 4 & 0 & 331 & 1 & 0 \\
5 & 14 & 0 & 0 & 9 & 0 & 0 & 75 & 0 & 0 \\
6 & 0 & 0 & 0 & 0 & 0 & 0 & 1 & 0 & 0 \\
\hline $\mathrm{SP}$ & 7 & 0 & 0 & 10 & 1 & 0 & 0 & 0 & 0 \\
\hline
\end{tabular}

Table 3: Distribution of tetrahedra among the different configurations at each iteration. Threshold $=10 \%$

We tested all models with two different thresholds: 0.1 (i.e. a relative error of $10 \%$ ), and 0.05 (or a relative error of 5\%). Tables 3 and 4 summarize the results obtained in these two cases for the three models listed above.

These tables show the number of tetrahedra of each type found for each of the first three iterations of our algorithm, and for each of the example models. Notice that the first row corresponds to case zero, where no further subdivision is required. The last row in these tables indicates the number of Shönhardt prisms found in each iteration.

Notice that after the second subdivision, most of the tetrahedra that require further subdivision correspond to the cases in which the subdivision process produces fewer tetrahedra (refer to the discussion in Section 3.2). Nonetheless the discrepancy quickly decreases below the specified threshold, as shown in Tables 5 and 6 . In these tables INT designate the number of tetrahedra at the beginning of each iteration, and FNT the number of tetrahedra after one step of subdivision. NTwE shows the number of tetrahedra that exceed the threshold and must be subdivided. Finally, "max. error" and "av. error" columns show the maximum relative discrepancy in the model at the start of the iteration and the average of the relative error. 


\begin{tabular}{|c||ccc|ccc|ccc|}
\hline Case & \multicolumn{3}{|c|}{ heart1 } & \multicolumn{3}{c|}{ heart2 } & \multicolumn{3}{c|}{ liver } \\
& Iter. 1 & Iter. 2 & Iter. 3 & Iter. 1 & Iter. 2 & Iter. 3 & Iter. 1 & Iter.2 & Iter. 3 \\
\hline \hline 0 & 104 & 2152 & 7857 & 56 & 1860 & 9562 & 3534 & 23151 & 41687 \\
1 & 94 & 580 & 433 & 97 & 786 & 616 & 1741 & 4213 & 2552 \\
2a & 111 & 500 & 209 & 128 & 649 & 161 & 1714 & 3397 & 1758 \\
$2 \mathrm{~b}$ & 2 & 10 & 10 & 11 & 24 & 23 & 94 & 479 & 67 \\
$3 \mathrm{a}$ & 3 & 6 & 1 & 4 & 3 & 0 & 64 & 84 & 1 \\
$3 \mathrm{~b}$ & 407 & 477 & 28 & 291 & 616 & 16 & 1971 & 187 & 28 \\
$3 \mathrm{c}$ & 37 & 110 & 23 & 52 & 145 & 9 & 424 & 178 & 67 \\
$4 \mathrm{a}$ & 65 & 24 & 3 & 103 & 7 & 0 & 430 & 21 & 5 \\
$4 \mathrm{~b}$ & 142 & 158 & 18 & 163 & 231 & 4 & 509 & 68 & 41 \\
5 & 42 & 23 & 0 & 76 & 11 & 0 & 440 & 2 & 0 \\
6 & 2 & 0 & 0 & 7 & 0 & 0 & 34 & 0 & 0 \\
\hline $\mathrm{SP}$ & 6 & 1 & 0 & 8 & 30 & 0 & 0 & 0 & 0 \\
\hline
\end{tabular}

Table 4: Distribution of tetrahedra among the different configurations at each iteration. Threshold $=5 \%$

Also notice that for the chosen thresholds, it is reasonable that the average errors to stop decreasing as shown.

In order to visualize the meaning of these discrepancies, Figures 15 and 16 show perfusion levels at two slices of a heart model. In each case, subfigure (a) displays the original perfusion levels stored in a voxel model, and subfigures (b) the linear interpolation with the initial tetrahedral mesh. In subfigures (c) we show the result after four iterations of our algorithm with a threshold of $10 \%$, and in (d) the result after four iterations for a threshold of 5\%. Notice that the subfigures (d) are in both cases very similar to the original (in (a)), showing the quick convergence of the algorithm. The extreme right subfigures show the discrepancies between (a) and (d), scaled by a factor of 20 in figure 15 and by a factor of 15 in figure 16 .

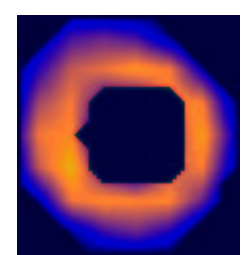

(a)

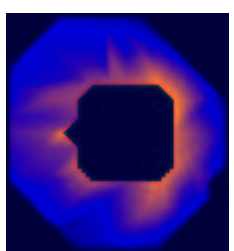

(b)

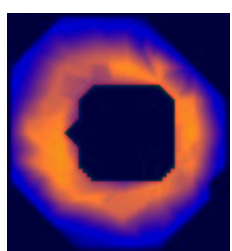

(c)

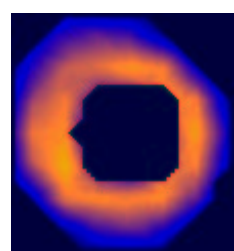

(d)

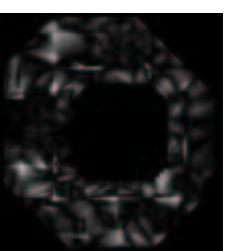

(e)

Figure 15: Perfusion levels of a heart's slice. (a) Original, (b) Original tetrahedral mesh, (c) Threshold=10\%, (d) Threshold $=5 \%$, (e) Scaled discrepancies by a factor of 20 


\begin{tabular}{|cc||c|c|c|c|c|}
\hline & & INT & FNT & NTwE & max. error & av. error \\
\hline \hline \multirow{3}{*}{ heart1 } & Iter. 1 & 1009 & 3176 & 728 & 0.471722 & 0.179860 \\
& Iter. 2 & 3176 & 4496 & 723 & 0.259945 & 0.072018 \\
& Iter. 3 & 4496 & 4554 & 44 & 0.183811 & 0.049100 \\
& Iter. 4 & 4554 & 4566 & 10 & 0.138826 & 0.048729 \\
\hline \multirow{3}{*}{ heart2 } & Iter. 1 & 988 & 3263 & 744 & 0.530826 & 0.199676 \\
& Iter. 2 & 3263 & 4043 & 519 & 0.257719 & 0.071939 \\
& Iter. 3 & 4043 & 4116 & 53 & 0.235371 & 0.061403 \\
& Iter. 4 & 4116 & 4162 & 30 & 0.184703 & 0.060667 \\
\hline \multirow{2}{*}{ liver } & Iter. 1 & 10955 & 20482 & 3648 & 0.540841 & 0.107953 \\
& Iter. 2 & 20482 & 25552 & 3333 & 0.443069 & 0.061281 \\
& Iter. 3 & 25552 & 26613 & 813 & 0.239975 & 0.050338 \\
& Iter. 4 & 26613 & 26836 & 171 & 0.166551 & 0.048441 \\
\hline
\end{tabular}

Table 5: Evolution of discrepancies at each iteration $($ Threshold $=10 \%)$

\begin{tabular}{|cc||c|c|c|c|c|}
\hline & & INT & FNT & NTwE & max. error & av. error \\
\hline \hline \multirow{3}{*}{ heart1 } & Iter. 1 & 1009 & 4040 & 905 & 0.471772 & 0.179860 \\
& Iter. 2 & 4040 & 8592 & 1888 & 0.322032 & 0.060891 \\
& Iter. 3 & 8592 & 9757 & 725 & 0.156437 & 0.028232 \\
& Iter. 4 & 9757 & 10047 & 180 & 0.138826 & 0.025605 \\
\hline \multirow{3}{*}{ heart2 } & Iter. 1 & 988 & 4342 & 932 & 0.530826 & 0.199676 \\
& Iter. 2 & 4342 & 10391 & 2482 & 0.257719 & 0.062552 \\
& Iter. 3 & 10391 & 11502 & 829 & 0.180630 & 0.032707 \\
& Iter. 4 & 11502 & 12033 & 331 & 0.154164 & 0.030557 \\
\hline \multirow{2}{*}{ liver } & Iter. 1 & 10955 & 31780 & 7421 & 0.540841 & 0.107953 \\
& Iter. 2 & 31780 & 46206 & 8629 & 0.497772 & 0.045310 \\
& Iter. 3 & 46206 & 53060 & 4519 & 0.210582 & 0.032588 \\
& Iter. 4 & 53060 & 56605 & 2382 & 0.168926 & 0.029993 \\
\hline
\end{tabular}

Table 6: Evolution of discrepancies at each iteration (Threshold $=5 \%)$ 


\begin{tabular}{|c||c|c|c|}
\hline & Initial quality & thrshld. 10\% & thrshld. 5\% \\
\hline \hline heart1 & 0.565231 & 0.578719 & 0.574900 \\
heart2 & 0.499718 & 0.508499 & 0.507415 \\
liver & 0.333292 & 0.313060 & 0.309224 \\
\hline
\end{tabular}

Table 7: Quality of the meshes

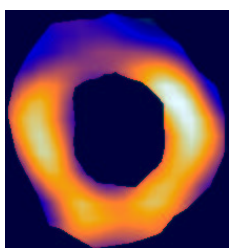

(a)

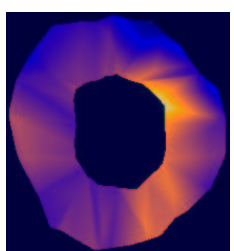

(b)

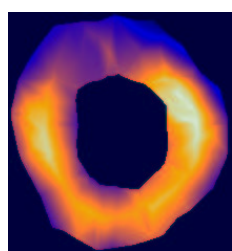

(c)

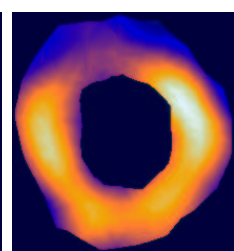

(d)

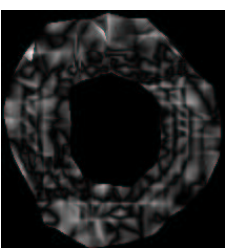

(e)

Figure 16: Perfusion levels of a heart's slice. (a) Original, (b) Original tetrahedral mesh, (c) Threshold=10\%, (d) Threshold=5\%, (e) Scaled discrepancies by a factor of 15

Finally, we conducted an experiment to measure the quality of the resulting tetrahedra. We measure the quality of the tetrahedra in a standard way, using the mean ratio, defined by Liu and Joe [8], that has the advantage of being invariant under traslation, rotation and uniform scaling, and is efficient to compute. For a tetrahedron $\mathscr{T}$, its quality is defined as $\eta=12(3 v)^{2 / 3} / \sum_{0 \leq i<j<3} l_{i j}^{2}$, where $v$ is the volume of $\mathscr{T}$ and $l_{i j}^{2}$ are the lengths of their edges. This measure is always a number between 0 and 1 , where 1 corresponds to a regular tetrahedron.

Our last Table 7, shows the resulting qualities for the two threshold values used in our experiments. Notice that in all cases the final qualities are similar (within $8 \%$ ) to the initial qualities. This is all that can be expected of an algorithm designed to minimize subdivision.

\section{Conclusions}

A method of adaptive subdivision has been developed to refine a tetrahedral mesh immersed in a voxel model (and which inherits from the voxel model the property values stored at its vertices) until the discrepancy of the volume property of interest computed from the tetrahedral mesh and from the original volume data is below a user-specified threshold.

The subdivision process is local, and requires no propagation from one cell to another. As such, each iteration is computed in a single sweep of the model, and 
requires no backtracking. Also for this reason, it does not require any additional information about the connectivity of the tetrahedral mesh to be stored.

If the initial mesh is conformal, the algorithm produces a conformal mesh as a result. Although the algorithm is local, its behavior on a facet of a tetrahedron depends solely on the information within that facet, and not the rest of the tetrahedron, which ensures that neighboring tetrahedra will be handled in a consistent way automatically.

The algorithm has shown a fast convergence to the desired discrepancy level in all models tested. 


\section{Bibliography}

[1] R. Bank, A. Sherman, and A. Weiser. Refinement algorithm and data structures for regular local mesh refinement. Scientific Computing, 44:3-17, 1983.

[2] J. Bey. Tetrahedral grid refinement. Computing, 55(4):355-378, 1995.

[3] D. Bielser and M. Gross. Interactive Simulation of Surgical Cuts. In Proc. of Pacific Graphics, pages 116-125. IEEE CS Press, 2000.

[4] E. Danovaro, L. De Floriani, M. Lee, and H. Samet. Multiresolution tetrahedral meshes: an analysis and a comparison. In Proc. Intern. Conference on Shape Modeling, pages 83-91. IEEE CS Press, 2002.

[5] C. Forest, H. Delingette, and N. Ayache. Cutting simulation of manifold volumetric meshes. In MICCAI, volume 2489 of LNCS, pages 235-244. SpringerVerlag, 2002.

[6] F. Ganovelli, P. Cignoni, C. Montani, and R. Scopigno. Enabling cuts on multiresolution representation. In Computer Graphics Intern., pages 183190. IEEE CS Press, 2000.

[7] G. Greiner and R. Grosso. Hierarchical tetrahedral-octaedral subdivision for volume visualization. The Visual Computer, 16(6):357-369, 2000.

[8] A. Liu and B. Joe. On the shape of tetrahedra from bisection. Mathematics of Computation, 63(207):141-154, 1994.

[9] A. Liu and B. Joe. Quality local refinement of tetrahedral meshes based on bisection. SIAM Journal on Scientific Computing, 16(6):1269-1291, 1995.

[10] A. Liu and B. Joe. Quality local refinement of tetrahedral meshes based on 8-subtetrahedron subdivision. Mathematics of Computation, 65(215):11831200, 1996.

[11] A. Mor and T. Kanade. Modifying soft tissue models: Progressive cutting with minimal new element creation. In MICCAI, volume 1935 of LNCS, pages 598-607. Springer-Verlag, 2000. 
[12] A. Plaza and G. Carey. About local refinement of tetrahedral grids based on bisection. In Proc. Intern. Meshing Roundtable, pages 123-136. Sandia Corporation, 1996.

[13] A. Plaza and G. Carey. Local refinement of simplicial grids based on skeleton. Applied Numerical Mathematics, 32:195-218, 2000.

[14] A. Plaza, M.A. Padrón, J.P. Suárez, and S. Falcón. The 8-tetrahedra longestedge partition of right-type tetrahedra. Finite Elements in Analysis and Design, 41:253-265, 2004.

[15] A. Plaza and M. C. Rivara. Mesh refinement based on the 8-tetrahedra longest-edge partition. In Proc. Intern. Meshing Roundtable, pages 67-78. Sandia Corporation, 2003.

[16] M.C. Rivara. Mesh refinement processes based on the generalized bisection of simplices. SIAM Journal on Numerical Analysis, 21(3):604-613, 1984.

[17] M.C. Rivara. Selective refinement/derefinement algorithms for sequences of nested triangulations. Intern. Journal for Numerical Methods in Engineering, 28:2889-2906, 1989.

[18] M.C. Rivara and G. Iribarren. The 4-Triangles longest-side partition of triangles and linear refinement algorithms. Mathematics of Computation, 65(216):1485-1502, 1996.

[19] M.C. Rivara and C. Levin. A 3d refinement algorithm suitable for adaptive and multigrid techniques. Communications in Applied Numerical Methods, 8:281-290, 1992.

[20] L. Rodríguez, I. Navazo, and Á. Vinacua. A tetrahedral model to represent the left ventricle volume of the heart. In Proc. Vision, Modeling and Visualization, pages 249-256. IOS Press, 2002.

[21] D. Ruprecht, R. Nagel, and H. Müller. Spatial free form deformation with scattered data interpolation methods. Computers \& Graphics, 19(1):63-71, 1995.

[22] Detlef Ruprecht and Heinrich Müller. A scheme for edge-based adaptive tetrahedron subdivision. In Visualization and Mathematics, pages 61-70. Springer Verlag, 1998.

[23] S. Zhang. Successive subdivisions of tetrahedra and multigrid methods on tetrahedral meshes. Houston Journal of Mathematics, 21(3):541-556, 1995.

[24] Y. Zhou, B. Chen, and A. Kaufman. Multiresolution tetrahedral framework for visualizing regular volume data. In Proc. IEEE Visualization conference, pages 135-142, 1997. 